\title{
AVALIAÇÃO DE CULTIVARES DE BERINJELA EM ARMAZENAMENTO REFRIGERADO
}

\author{
Ricardo Alfredo Kluge ${ }^{1}$; Antônio Carlos Carvalho Antonini²; Walter Geremias Rodriguez \\ Robles$^{1}$; João Tessarioli Neto'; Angelo Pedro Jacomino ${ }^{1 *}$; João Alexio Scarpare Filho' \\ ${ }^{1}$ Depto. de Produção Vegetal - ESALQ/USP, C.P. 9 - CEP: 13418-900 - Piracicaba, SP. \\ ${ }^{2}$ CATI, R. Cel. Procópio Carvalho, 676 - CEP: 13660-000 - Porto Ferreira, SP. \\ *e-mail: jacomino@carpa.ciagri.usp.br
}

RESUMO: Frutos de diferentes cultivares de berinjela foram armazenados a $11 \pm 0,5^{\circ} \mathrm{C}$ e $90 \pm 5 \%$ UR, por períodos de 7,14 e 21 dias (+ 2 dias a $24-25^{\circ} \mathrm{C}$ e $70-75 \%$ UR), com o objetivo de verificar o comportamento pós-colheita destes frutos. As cultivares estudadas foram Diamante Negro, Kiko, Piracicaba F-100, Flórida Market, Super F-100, F-2000, Suzuki, Nápoli e Ciça. Verificaram-se diferenças nas respostas das diferentes cultivares em relação à perda de peso, pressão de turgescência e qualidade global durante o armazenamento refrigerado. A frigoconservação dos frutos foi viável até 7 dias para todos as cultivares. O armazenamento por período superior a 7 dias dependeu da cultivar. A cultivar Super F-100 foi a que apresentou a maior capacidade de armazenamento (21 dias).

Palavras-chave: Solanum melongena, turgescência, perda de peso, murchamento

\section{POST-HARVEST QUALITY OF EGGPLANT CULTIVARS IN COLD STORAGE}

ABSTRACT: Our objective was to evaluate the post-harvest behavior of nine eggplant cultivars. Fruits were stored at $11 \pm 0,5^{\circ} \mathrm{C}$ e $90 \pm 5 \% \mathrm{RH}$, for periods of 7,14 and 21 days (including 2 days of simulated commercialization at $24-25^{\circ} \mathrm{C}$ and $\mathbf{7 0 - 7 5 \%} \mathrm{RH}$ ). The cultivars studies were Diamante Negro, Kiko, Piracicaba F-100, Flórida Market, Super F-100, F-2000, Suzuki, Nápoli and Ciça. We measured changes in weight loss, turgor pressure and visual quality during the cold storage period. Low temperature storage of the fruits was acceptable up to 7 days for all cultivars. Storage above 7 days was dependent of the cultivar. The cultivar Super F-100 presented the largest storage capacity.

Key words: Solanum melongena, turgor, weight loss, shriveling

\section{INTRODUÇÃO}

A refrigeração tem sido o método de conservação mais utilizado para a manutenção e o aumento da vida útil de frutas e hortaliças, por reduzir o metabolismo e a perda de peso (Chitarra \& Chitarra, 1990).

Berinjelas são frutos sensíveis às injúrias pelo frio, principalmente em temperaturas inferiores a $10^{\circ} \mathrm{C}$, apresentando, de acordo com o tempo de exposição, uma série de sintomas que depreciam a sua comerciabilidade (Salunkhe \& Desai, 1984). O aparecimento de depressões superficiais ("surface pitting"), bronzeamento da casca e escurecimento da polpa e das sementes, são os principais sintomas de injúrias pelo frio nesta hortaliça. Os frutos injuriados também sofrem maior incidência de podridões fúngicas, causadas principalmente por Alternaria sp. (Wills et al., 1981; Salunkhe \& Desai, 1984; Hardenburg et al., 1986).
A temperatura e umidade relativa do ar mais recomendadas para o armazenamento de berinjela são $10-13^{\circ} \mathrm{C}$ e $90-95 \%$, respectivamente. Nestas condições pode-se conservar os frutos até 3 semanas (Pantastico, 1975; Ryall \& Lipton, 1979; Hardenburg et al., 1986).

No Brasil, a berinjela é geralmente comercializada a granel e a frigorificação é raramente utilizada (Henz \& Silva, 1995). Após 3 a 4 dias em temperatura ambiente, a qualidade dos frutos é bastante reduzida, e estes apresentam-se murchos, esponjosos e sem brilho, o que reduz o seu valor comercial (Mohammed \& Sealy, 1988; Henz \& Silva, 1995). Assim, a refrigeração pode ser utilizada para reduzir as perdas pós-colheita, manter a qualidade, aumentar a vida útil do produto e, com isso, estender o período de comercialização desta importante hortaliça.

Vários fatores afetam a conservação de berinjelas, além das condições de armazenamento. A cultivar representa um desses 
fatores, pois cada genótipo apresenta sua taxa própria de deterioração, em função de suas características intrínsecas e tamanho de fruto (Uncini et al., 1977; Sigrist, 1984; Henz \& Silva, 1995). Recentemente Díaz-Pérez (1998) verificou, em berinjelas 'Classic', que o tamanho do fruto influenciou a perda de peso, sendo essa maior nos frutos menores, devido à maior área de superfície de exposição, em relação a sua massa, e maior porção do cálice cobrindo os frutos.

O murchamento é indesejável para frutos frescos, uma vez que afeta a sua aparência e, desta maneira, o seu valor de mercado. O murchamento é decorrente, principalmente, da transpiração do fruto, em resposta ao déficit de pressão de vapor (DPV) entre os seus tecidos e o ambiente externo. O DPV é governado tanto pela temperatura como pela umidade relativa do ar. Assim, quanto mais alta a temperatura e/ou mais baixa a umidade do ar, maior é a DPV, levando a uma maior perda de água do fruto para o ambiente (Grierson \& Wardowski, 1978; Hardenburg et al., 1986; Woods, 1990). Portanto, em condições ambientais, o DPV é superior ao verificado na refrigeração, e os frutos são mais afetados pelo murchamento. É importante, no caso de berinjelas e outros produtos sensíveis ao murchamento, que sua manipulação seja realizada em temperatura mais baixa que a do ambiente, e alta umidade relativa, para evitar perda excessiva de água dos frutos e ampliar o período de comercialização.

Com a criação de novas cultivares, mais adaptadas e produtivas, existe a necessidade de conhecer-se a capacidade de conservação das mesmas. $O$ objetivo do presente trabalho foi verificar o comportamento de nove cultivares de berinjela em armazenamento refrigerado.

\section{MATERIAL E MÉTODOS}

O experimento foi realizado no Departamento de Produção Vegetal da Escola Superior de Agricultura "Luiz de Queiroz", ESALQ/USP, em Piracicaba (SP). Frutos de nove cultivares de berinjela foram colhidas no período da manhã (8-10hs) do dia 12/01/98 e levados ao Laboratório de Pós-colheita. Para o experimento, selecionados frutos sadios e sem danos físicos. As seguintes cultivares foram utilizadas: Diamante Negro, Kiko, Piracicaba F-100, Flórida Market, Super F-100, F-2000, Suzuki, Nápoli e Ciça.
Os frutos foram armazenados em câmara fria a $11 \pm 0,5^{\circ} \mathrm{C}$ e $90 \pm 5 \%$ de umidade relativa, por períodos de 7, 14 e 21 dias. Após cada período de armazenamento, os frutos foram submetidos à temperatura $\left(24-25^{\circ} \mathrm{C}\right)$ e $70-75 \%$ UR, durante 2 dias, visando simular o período de comercialização.

O delineamento experimental foi inteiramente ao acaso para cada período de conservação, constando de nove tratamentos (nove cultivares) com quatro repetições. Foram utilizados seis frutos por repetição. Os resultados obtidos para as variáveis perda de peso e pressão de turgescência foram submetidos à análise da variância (teste F) e comparação de médias pelo teste de Tukey ao nível de $5 \%$ de probabilidade. Para a variável qualidade global testou-se inicialmente a homogeneidade das variâncias através do teste de Hartley. Como não se constatou heterogeneidade das variâncias ao nível de $a=0,05 \%$, procedeu-se os mesmos testes das outras variáveis. Para cada cultivar foi realizada análise de regressão para a variável qualidade global, com o objetivo de identificar a máxima capacidade de armazenamento.

Realizou-se avaliação inicial das cultivares utilizadas, determinando-se o peso, comprimento e pressão de turgescência, em 20 frutos de cada cultivar.

As variáveis analisadas foram: a) perda de peso: foi determinada pela diferença, em porcentagem, entre o peso inicial e o peso verificado ao final de cada período de armazenamento; b) pressão de turgescência: foi determinada pela técnica da aplanação, utilizando-se um aplanador horizontal, conforme método de Calbo \& Nery (1995). Os resultados foram expressos em kgf. $\mathrm{cm}^{-2}$; c) qualidade global: foi determinada a partir de uma escala subjetiva de notas variando de 0 a 3 , onde $3=$ boa qualidade; 2 = regular; 1 = ruim; $0=$ péssima. Para esta determinação, 5 avaliadores foram utilizados. Estes consideraram: frutos de boa qualidade (nota 3 ) = brilhosos e sem murchamento perceptível ao tato; frutos de qualidade regular (nota 2$)=$ sem brilho e com murchamento pouco perceptível ao tato; frutos de qualidade ruim (nota 1 ) = sem brilho e com murchamento perceptível ao tato; frutos de qualidade péssima (nota 0$)=$ sem brilho, com murchamento perceptível ao tato e com início de podridão. Considerou-se que as notas 3 e 2 constituíam frutos aptos a serem comercializados, e as notas 1 e 0 representavam frutos impróprios para a comercialização. 


\section{RESULTADOS E DISCUSSÃO}

Observaram-se diferenças entre as cultivares quanto ao peso, comprimento médio e pressão de turgescência dos frutos na avaliação realizada imediatamente após a colheita (TABELA 1).

Houve variação para a perda de peso dos frutos entre cultivares, durante 0 armazenamento refrigerado (Figura 1). Aos 7 dias de conservação frigorificada $(+2$ dias à temperatura ambiente) foi observada, para a cultivar 'Suzuki', a maior perda de peso (12,01\%), diferindo significativamente de 'Super F-100' $(7,61 \%)$, que por sua vez não diferiu das demais cultivares. $\mathrm{Na}$ avaliação realizada aos 14 dias, a cultivar 'Kiko' apresentou a maior perda de peso $(18,55 \%)$, diferindo estatisticamente de 'Piracicaba F-100' (12,03\%) e 'Super F-100' $(11,89 \%)$. Essas cultivares, por sua vez, não diferiram das demais. Aos 21 dias, não houve diferença significativa para a perda de peso nas culti-vares testadas. Neste período, a perda de peso oscilou de 15,49\% ('Diamante Negro') a 21,98\% ('Kiko').

A elevada perda de água, verificada aos 21 dias de armazenamento, resultou em murchamento perceptível em todas as cultivares testadas, influenciando negativamente a qualidade das mesmas.

Quanto a pressão de turgescência que, segundo Calbo \& Nery (1995), está relacionada com a firmeza do fruto, houve diferenças significativas entre as cultivares testadas (Figura 2). Estas diferenças podem estar relacionadas
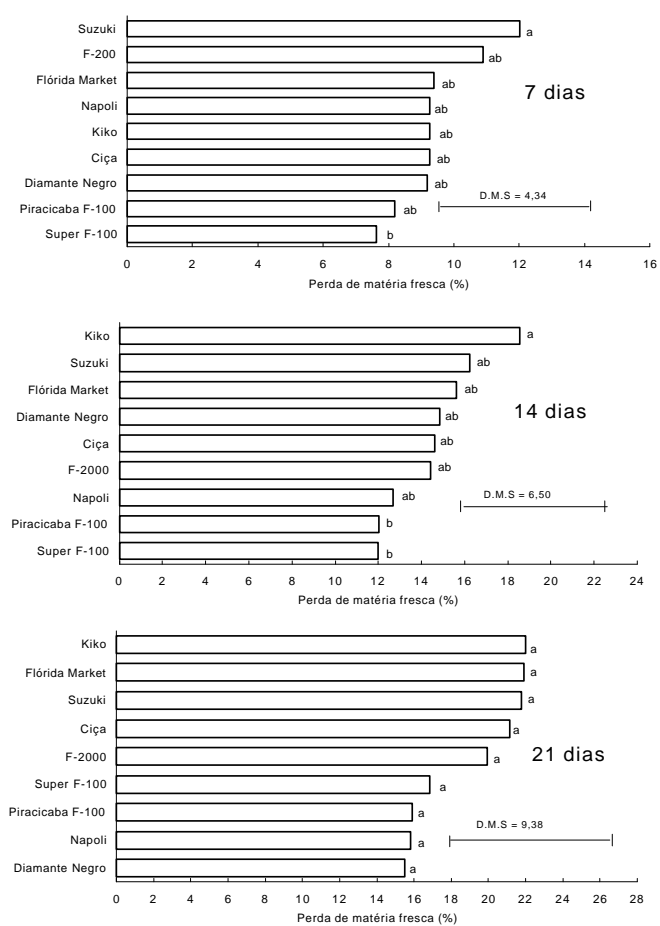

Figura 1 - Perda de peso (\%) de diferentes cultivares de berinjela armazenadas a $11 \pm 0,5^{\circ} \mathrm{C}$ e $90-95 \%$ UR ( 2 dias a $24-25^{\circ} \mathrm{C}$ e $70-75 \%$ UR), por $7,14 \mathrm{e}$ 21 dias. Médias seguidas de mesma letra não diferem entre si pelo teste de Tukey ao nível de $5 \%$ de probabilidade.

TABELA 1 - Peso, comprimento e pressão de turgescência em frutos recém colhidos de nove cultivares de berinjela utilizadas no experimento.

\begin{tabular}{lccc}
\hline Cultivar & Peso do fruto $(\mathrm{g})$ & Comprimento do fruto $(\mathrm{cm})$ & Pressão de turgescência $\left(\mathrm{kgf.cm}^{-2}\right)$ \\
\hline Diamante Negro & $245 \mathrm{bcd}$ & $15,1 \mathrm{a}$ & $0,58 \mathrm{abc}$ \\
Kiko & $305 \mathrm{a}$ & $14,3 \mathrm{a}$ & $0,53 \mathrm{bc}$ \\
Piracicaba F-100 & $269 \mathrm{abc}$ & $13,8 \mathrm{a}$ & $0,51 \mathrm{bc}$ \\
Flórida Market & $260 \mathrm{abcd}$ & $13,5 \mathrm{a}$ & $0,69 \mathrm{a}$ \\
Super F-100 & $290 \mathrm{ab}$ & $15,3 \mathrm{a}$ & $0,62 \mathrm{ab}$ \\
F-2000 & $212 \mathrm{de}$ & $14,9 \mathrm{a}$ & $0,61 \mathrm{abc}$ \\
Suzuki & $163 \mathrm{e}$ & $14,7 \mathrm{a}$ & $0,69 \mathrm{a}$ \\
Nápoli & $243 \mathrm{bcd}$ & $14,3 \mathrm{a}$ & $0,49 \mathrm{c}$ \\
Ciça & $227 \mathrm{~cd}$ & $15,0 \mathrm{a}$ & $0,60 \mathrm{abc}$ \\
\hline C.V. $(\%)$ & 7,27 & 8,78 & 7,41 \\
\hline
\end{tabular}

Médias seguidas de mesma letra na coluna não diferem entre si pelo teste de Tukey ao nível de $5 \%$ de probabilidade. 
com o valor inicial de turgescência, que variou conforme a cultivar (TABELA 1). De maneira geral, os frutos que se mostraram mais firmes à época da colheita tenderam a manter-se com maior pressão de turgescência durante a conservação. Fazendo-se um cálculo percentual da perda de pressão de turgescência ao longo dos períodos estudados, em relação à avaliação de colheita, observou-se comportamento semelhante para as cultivares estudadas, tendo sido verificada redução de $50 \%$, em média, da pressão de turgescência aos 7 dias, de 60 a $70 \%$ aos 14 dias, e 70 a $80 \%$ aos 21 dias.

A qualidade global dos frutos durante 0 armazenamento variou significativamente entre as cultivares, nos três períodos de arma-
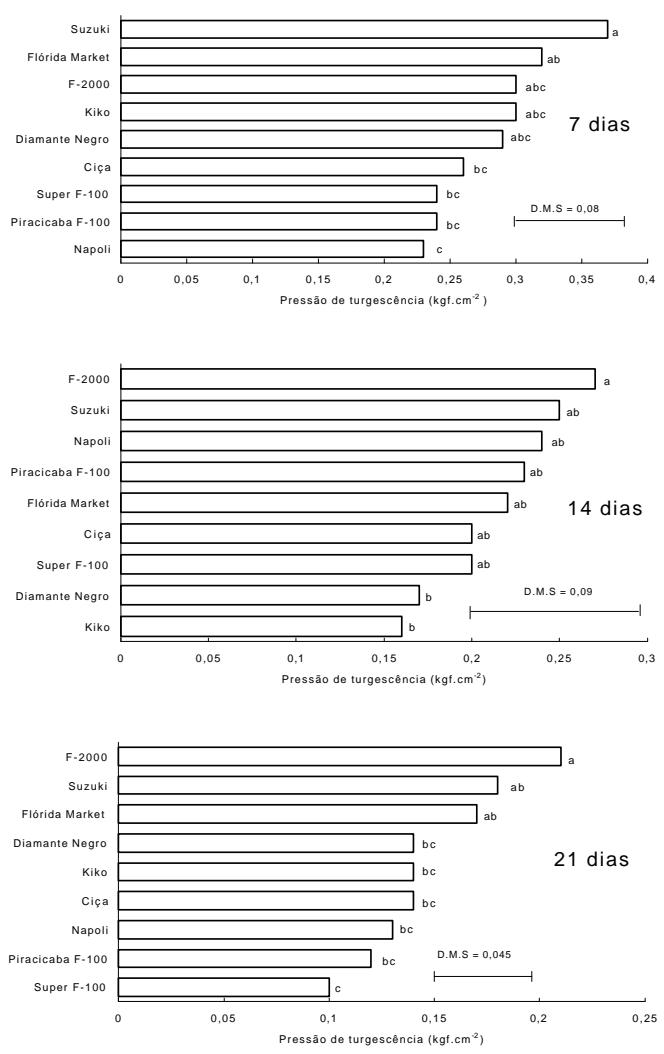

Figura 2 - Pressão de turgescência $\left(\mathrm{kgf.cm}^{-2}\right)$ de diferentes cultivares de berinjela armazenadas a $11 \pm 0,5^{\circ} \mathrm{C}$ e $90-95 \%$ UR (+ 2 dias a $24-25^{\circ} \mathrm{C}$ e $70-75 \%$ UR), por 7,14 e 21 dias. Médias seguidas de mesma letra não diferem entre si pelo teste de Tukey ao nível de $5 \%$ de probabilidade. zenamento estudados (Figura 3). Aos 7 dias de armazenamento, 'Piracicaba F-100' e 'Super F-100' apresentaram qualidade global signifi-cativamente superior à 'Suzuki' que, por sua vez, não diferiu das demais.

Aos 14 dias, houve queda generalizada na qualidade dos frutos, principalmente para 'Diamante Negro', 'Suzuki', 'Flórida Market' e 'Kiko', que já apresentavam murchamento perceptível. A cultivar Kiko apresentou a pior qualidade global e a maioria dos frutos estavam impróprios para a comercialização. 'Piracicaba F-100', 'Super F-100', 'Nápoli', 'Ciça' e 'F-2000' apresentaram-se com qualidade regular aos 14 dias de armazenamento e com início de murchamento, mas ainda comercializáveis.
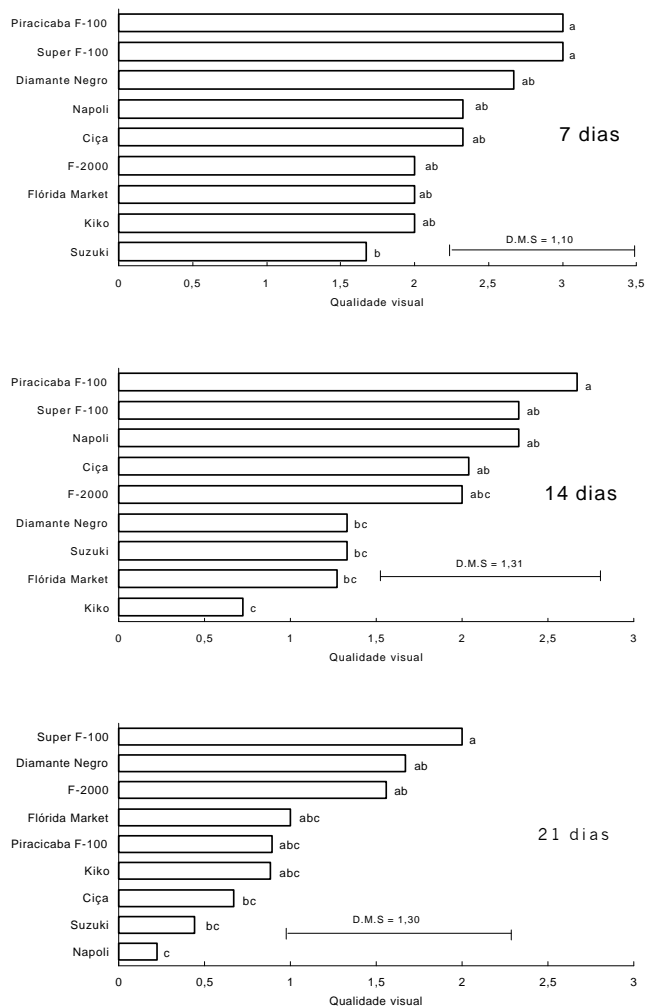

Figura 3 - Qualidade global de diferentes cultivares de berinjela armazenadas a $11 \pm 0,5^{\circ} \mathrm{C}$ e $90-95 \%$ UR (+ 2 dias a $24-25^{\circ} \mathrm{C}$ e $70-75 \%$ UR), por 7,14 e 21 dias. (Notas: 3 = boa qualidade; 2 = regular; 1 = ruim; 0 = péssima). Médias seguidas de mesma letra não diferem entre si pelo teste de Tukey ao nível de $5 \%$ de probabilidade. 
Aos 21 dias, houve queda ainda maior na qualidade global para todas as cultivares. A cultivar 'Super F-100' apresentou melhor qualidade, embora seus frutos apresentassem início de murchamento perceptível. As demais cultivares apresentaram baixa qualidade neste período, devido ao elevado grau de murchamento e início de podridão.

Efetuando-se a análise de regressão para a variável qualidade global pôde-se observar a capacidade máxima aproximada de armazenamento das cultivares testadas (Figura 4). Esta máxima capacidade foi identificada quando a qualidade global atingiu a nota 2 , que correspondeu à qualidade regular, com os frutos apresentando-se sem brilho e com início de murchamento, mas ainda em condições de serem comercializados. Assim, verificou-se que, para as condições de armazenamento empregadas do experimento, as cultivares apresentaram as seguintes capacidades máximas de armazenamento: Super F-100 = 21 dias; Piracicaba F-100 = 17 dias; Nápoli = 14 dias; Diamante Negro e F-2000 = 13 dias; Ciça = 11 dias; Kiko $=8$ dias; Flórida Market e Suzuki = 7 dias (Figura 4). No presente trabalho observou-se comportamentos diferenciados entre as cultivares de berinjela sob armazenamento refrigerado, concordando com observações prévias de outros autores, os quais relataram que a capacidade de conservação desta hortaliça sofre variação de acordo com a cultivar, devido às distintas taxas metabólicas e características físicas dos frutos (Uncini et al., 1977; Sigrist, 1984; Henz \& Silva, 1995; Díaz-Pérez, 1998).

Nas condições em que os frutos foram armazenados, observou-se grande perda de peso e de turgescência nos períodos de armazenamento estudados, o que reduziu significativamente a qualidade do produto. $O$ período de dois dias de comercialização, utilizado no presente trabalho, serviu para simular o tempo mínimo aproximado em que os frutos são retirados do armazenamento, embalados, transportados e comercializados. Neste período, os frutos permaneceram em condições ambientais, contribuindo para a elevação na perda de peso e de turgescência, devido à maior temperatura e menor umidade relativa do ar.

Com berinjelas, estudos ainda são necessários visando reduzir as perdas pós-colheita
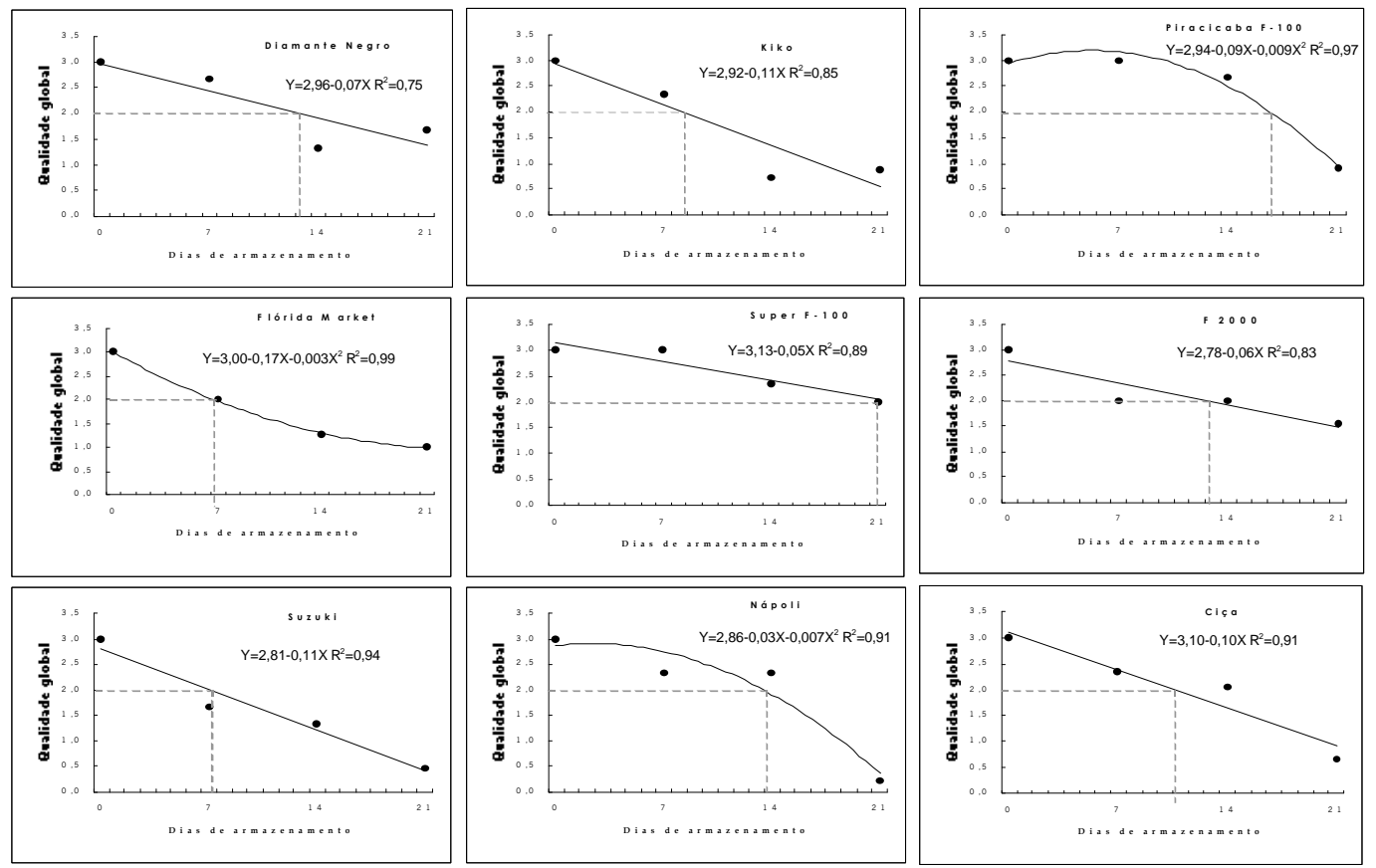

Figura 4 - Análise de regressão para a variável qualidade global de diferentes cultivares de berinjela armazenadas a $11 \pm 0,5^{\circ} \mathrm{C}$ e $85-90 \%$ UR (+ 2 dias a $24-25^{\circ} \mathrm{C}$ e $60-70 \%$ UR), por 7,14 e 2 dias. A máxima capacidade de armazenamento foi definida pela nota 2 (frutos com qualidade regular, sem brilho e com pouco murchamento). 
e aumentar a capacidade de conser-vação e comercialização desta hortaliça. Dentre os fatores que justificam tais estudos está o aumento de cultivares disponíveis aos pro-dutores. A criação de novas cultivares, mais produtivas e de melhor qualidade, deve ser acompanhada de estudos relacionados ao armazenamento refrigerado e à vida de prateleira dos frutos, para determinar seu período de comercialização.

\section{CONCLUSÕES}

Para as condições de armazenamento de $11 \pm 0,5^{\circ} \mathrm{C}$ e $90-95 \%$ UR $\left(+2\right.$ dias a $24-25^{\circ} \mathrm{C}$ e $70-75 \%$ UR), as seguintes conclusões podem ser efetuadas:

- Diferentes cultivares de berinjela apresentam comportamento diferenciado durante o armazenamento refrigerado;

- As cultivares testadas apresentam as seguintes capacidades máximas de armazenamento: Super F-100 $=21$ dias; Piracicaba F-100 = 17 dias; Nápoli $=14$ dias; Diamante Negro e F-2000 = 13 dias; Ciça $=11$ dias; Kiko = 8 dias; Flórida Market e Suzuki $=7$ dias.

\section{REFERÊNCIAS BIBLIOGRÁFICAS}

CALBO, A.G.; NERY, A.A. Medida de firmeza em hortaliças pela técnica da aplanação. Horticultura Brasileira, v.12, n.1, p.14-18, 1995.

CHITARRA, M.I.F.; CHITARRA, A.B. Pós-colheita de frutos e hortaliças: fisiologia e manuseio. Lavras: ESAL/FAEPE, 1990. 320p.

DÍAZ-PÉREZ, J.C. Transpiration rates in eggplant fruit as affected by fruit and calyx size. Postharvest Biology and Technology, v.13, n.1, p.45-49, 1998.

GRIERSON, W.; WARDOWSKI, W.F. Relative humidity effects on the postharvest life in fruits and vegetables. HortScience, v.13, n.5, p.22-26, 1978.
HARDENBURG, R.E.; WATADA, A.E.; WANG, C.Y. The commercial storage of fruits, vegetables and florist, and nursery stocks. USDA: Washington, 1986. 130p.

HENZ, G.P.; SILVA, C. Conservação de frutos de berinjela cv. Ciça através de refrigeração e embalagem. Pesquisa Agropecuária Brasileira, v.30, n.2, p.157-162, 1995.

MOHAMMED, M; SEALY, L. Hidrocooling and postharvest quality in melongene (Solanum melongena L.). Tropical Agriculture, v.65, n.2, p.161-165, 1988.

PANTASTICO, E. B. Postharvest physiology, handling and utilization of tropical and subtropical fruits and vegetables. Westport: AVI, 1975. $559 \mathrm{p}$.

RYALL, A.L.; LIPTON, W.J. Handling, transportation and storage of fruits and vegetables. 2 ed. Westport: AVI, 1979. 560p.

SALUNKHE, D.K.; DESAI, B.B. Postharvest biotechnology of vegetables. Boca Raton: CRC Press, 1984. 194p. v.2.

SIGRIST, J.M.M. Taxa de deterioração, respiração e produção de etileno de diferentes cultivares de berinjela (Solanum melongena). Coletânea do ITAL, v.14, p.117-134, 1984.

UNCINI, L.; GORINI, F.; SOZZI, A. Agronomic value and cold-keeping of eggplant (Solanum melongena) varieties and hybrids. Acta Horticulturae, n.58, p.383-388, 1977.

WILLS, R.H.H.; LEE, T.H.; GRAHAM, W.B.; HALL, E.G. Postharvest: an introduction to the physiology and handling of fruit and vegetables. Kensington: New South Wales University Press, 1981. 161p.

WOODS, J.L. Moisture loss from fruits and vegetables. Postharvest News and Information, v.1, n.3, p.195-199, 1990.

Recebido para publicação em 15.03 .99

Aceito para publicação em 16.07.99 